\title{
Microwave Enhanced Roasting for Pyrite Ore Samples with Dielectric Properties Strongly Dependent on Temperature ${ }^{1}$
} Tostación mejorada con microondas en muestras piríticas con propiedades dieléctricas marcadamente dependientes

\author{
de la temperatura ${ }^{2}$
}

\author{
Manuel Díaz ${ }^{3}$ \\ Iván Amaya ${ }^{4}$ \\ Rodrigo Correa
}

doi:10.11144/Javeriana.jyu20-1.merp

How to cite this article:

M. Díaz, I. Amaya, and R. Correa, "Microwave enhanced roasting for pyrite ore samples with dielectric properties strongly dependent on temperature," Ing. Univ., vol. 20, no. 1, pp. 63-84, 2016. http://dx.doi.org/10.11144/Javeriana.iyu20-1.merp

\footnotetext{
' Scientific and technological research article. Submitted on: November 19th, 2014. Accepted on: August 14th, 2015. This article is derived from Calentamiento volumétrico de nanolechos para la recuperación de trazas de metales preciosos presentes en minerales $y$ sus colas, research project, with code 5551. This project was carried out within the research group CEMOS at Universidad Industrial de Santander, Bucaramanga, Colombia.

${ }^{2}$ Artículo de investigación científica y tecnológica. Fecha de recepción: 19 de noviembre de 2014. Fecha de aceptación: 14 de agosto de 2015. Este artículo se deriva de un proyecto de investigación denominado Calentamiento volumétrico de nanolechos para la recuperación de trazas de metales preciosos presentes en minerales y sus colas, código 5551, y desarrollado por el grupo de investigación CEMOS de la Universidad Industrial de Santander, Bucaramanga, Colombia. ${ }^{3}$ Electronic Engineer, Universidad Industrial de Santander, Bucaramanga, Colombia. E-mail: manfer_579@hotmail.com ${ }^{4}$ Mechatronic Engineer, Universidad Autónoma de Bucaramanga, Bucaramanga, Colombia. PhD (c) on Engineering, Universidad Industrial de Santander, Bucaramanga, Colombia. E-mail: ivan.amaya2@correo.vis.edu.co

${ }^{5}$ Chemical Engineer, Universidad Nacional de Colombia. MSc on Chemical Engineering, Lehigh University. MSc on Chemical Engineering, Universidad Industrial de Santander, Bucaramanga, Colombia. PhD on Polymer Science and Engineering, Lehigh University. Professor, Escuela de Ingenierías Eléctrica, Electrónica y de Telecomunicaciones, Universidad Industrial de Santander. E-mail: crcorrea@uis.edu.co
} 


\section{Abstract}

This article shows the main experimental results related to measuring dielectric properties of Pyrite ore mineral samples as a function of temperature, and their effect on the heating behavior of the samples. It was found that the real, $\epsilon^{\prime}(T)$, and imaginary, $\epsilon^{\prime \prime}(T)$, components of permittivity are strongly dependent on temperature. The model that best fitted experimental data for $\epsilon^{\prime}(T)$ and $\epsilon^{\prime}(T)$, was a Gaussian correlation, reaching $R^{2}$ values over 0.995 . Besides, and under certain conditions, it was possible to roast the mineral even better than with an electric furnace, while requiring less processing time (about $77.5 \%$ less) and with lower electrical energy consumption (about $80.8 \%$ less). Additional exploratory tests revealed that microwaves can be used to smelt a roasted mineral ore with time reductions of about $90 \%$, while keeping recovery margins above $95 \%$. Next stages of research should be directed to using a single mode applicator for processing higher volumes of mineral at pilot plant scale.

\section{Keywords}

roasting; gold; mineral ore; microwave heating; smelting

\section{Resumen}

Este artículo muestra los principales resultados experimentales relacionados con la medición de propiedades dieléctricas de muestras minerales con pirita, en función de la temperatura, así como su efecto en el calentamiento de las muestras. Se encontró que las componentes real, $\epsilon^{\prime}(T)$, e imaginaria, $\epsilon^{\prime \prime}(T)$, de la permitividad dependen fuertemente de la temperatura. El mejor modelo, para $\epsilon^{\prime}(T)$ y $\epsilon^{\prime \prime}(T)$, que se ajustó a los datos experimentales fue un modelo gaussiano, con valores de $R^{2}$ mayores a 0,995 . Además, y bajo ciertas condiciones, fue posible tostar el mineral incluso mejor que con un horno eléctrico, pues requirió menos tiempo de procesamiento (aproximadamente $77,5 \%$ menos), y con menor consumo de energía eléctrica (aproximadamente 80,8\% menos). Pruebas exploratorias adicionales revelaron que las microondas pueden ser utilizadas para fundir un mineral tostado, con reducciones de tiempo cercanas al $90 \%$ y manteniendo los márgenes de recuperación sobre el $95 \%$. Las etapas siguientes de investigación deben dirigirse a utilizar un aplicador monomodo, para procesar mayores volúmenes de mineral a escala de planta piloto.

\section{Palabras clave}

tostación; oro; mineral; calentamiento microondas; fundición 


\section{Introduction}

In the field of microwave-assisted processing the interaction between electromagnetic field and the Material Under Test (MUT) is well established [1]. The resulting effect is strongly dependent, among other variables, on the complex electrical permittivity and magnetic permeability of the sample, as well as on the geometry relation between the microwave applicator and the MUT. Still, this does not imply that a direct scaling of the problem can be carried out to pilot plant or industrial levels, since electromagnetic field distribution relates to the size of the microwave applicator. In that sense, it is vital to carry out preliminary lab-scale experiments to determine the interaction strength between materials (minerals in our study) and electromagnetic fields, becoming an obligated starting point when scaling the process.

Efforts to expand the use of microwaves as a heating method have been made around the world. Lately, several researches have reported experimental results, at lab scale, dealing with the interaction between microwave and mineral ores. They found, for example, that most sulfides, sulfosalts, and arsenides strongly interacted with microwaves and were rapidly heated [2]. Now, there are certain groups of minerals classified as poor and good microwave absorbers. This increasing interest seems to be due to the great pressure to achieve energy savings and clean production in the mineral processing and extractive metallurgical new industries [3]-[8].

Recent studies in the last decade, such as [9], showed that a microwaveassisted thermal treatment of ores reduced the energy required to grind the sample. The authors used samples with a weak interaction with microwaves (e.g. Quartz and Barite), and samples with a strong interaction (e.g. Pyrite, Chalcopyrite, Magnesite, Galenite, and Siderite). They heated the minerals using a source power of $900 \mathrm{~W}$ for a time interval between 1-5 min, using the ISM-approved frequency band of $2.45 \mathrm{GHz}$. The authors also simulated microwave interaction using commercial software to determine temperature distribution inside Barite and Magnesite samples. However, they considered electromagnetic and 
physical properties (i.e. density, conductivity, specific heat, dielectric constant, etc.) provided by the software, for a given electric field and operating frequency. In contrast, during this study a report is given on the variation of dielectric properties as a function of temperature, using experimental data. The current manuscript also presents the way in which microwave absorption by a sample can vary if the particle size is too small.

Peltosaari et al., carried out heat treatment experiments with a domestic microwave furnace using $\mathrm{SiC}$ as a thermal enhancer, and comparing against data for a conventional furnace heated by a resistance. They showed that within the microwave furnace, phase transformation of spodumene began after $110 \mathrm{~s}$ of heating, and samples were converted almost completely to $\beta$-spodumene after $170 \mathrm{~s}$. They observed that the grain size did not affect significantly the conversion rate, and stated that heating in microwave furnace for $170 \mathrm{~s}$ corresponded with the heating of approximately $480-600 \mathrm{~s}$ at temperature of $1100{ }^{\circ} \mathrm{C}$ in the conventional furnace. Also, Peltosaari and coworkers validated the conversion of spodumene samples with X-ray diffraction (XRD) and with field emission scanning electron microscope (FESEM) [10].

In a similar fashion, Bobicki et al. reported on the heating effect of using microwaves on ultramafic nickel ores [11]. They presented a process where serpentine was transformed into olivine due to high temperatures easily obtained with microwaves for this kind of material. They also showed that microwaves are a feasible approach for transforming a valuable Nickel material (Pentlandite) to other mineral forms where a compound made of Iron, Nickel, and Sulfur is present. Zhao et al. exploited the characteristic benefit of microwaves (i.e. selective heating) to treat Panzhihua Ilmenite ores (China) [12]. They achieved a highly-efficient process with low energy consumption, while preserving (and sometimes improving) the properties of the original Ilmenite. Using SEM analysis, they visualized cracks along the grain boundaries after microwave exposure. The authors concluded that these cracks were due to thermal stress, generated because of the different microwave interaction strengths of gangue minerals (weak effect) and of Ilmenite (strong eExperimental microwave processing and its modeling requires knowing the dielectric properties of the MUT as a function of temperature. In that sense, this article presents the main experimental results with some samples that can be compared against the data found by Lovás et al. [13]. They dealt with the influence of temperature on the dielectric properties of Pyrite, Galena, Chalcopyrite, Magnesite, Siderite, and Andesite, taken from different parts of the Slovak Republic. 
Interaction of microwaves does not limit to temperature increments to generate chemical reactions, as they also include mechanical effects. For example, a calcite matrix (low microwave interaction) that contains small amounts of Pyrite (high microwave interaction) was used as a model by Wang and Djordjevic [14]. They used a thermal breakage process and FEM to model thermal stress generation that leads to internal cracking in the ore. They estimated the presence of a large thermal stress around the interphase region between the continuous and discrete phases. However, they explained that the whole mineral fracture does not start in this region, but at a point located some distance away, and due to thermal expansion stresses. Unfortunately, they do not present an experimental validation of their data.

Aside from its scientific interest, gold has an important role in our society, whether for cosmetic or for industrial means. However, not all gold deposits can be easily processed, and unfortunately in some cases they require chemical reagents such as Mercury and Cyanide. In fact, they could also need some extra stages, such as grinding and gravimetric concentration, plus the ones required for the use of the reagents (such as pools). Additionally, each stage requires supervision to avoid leakages, and to prevent damaging the ecosystems. All of this increases processing time and costs, so new alternatives should be explored. This research shows the varying dielectric properties of a Pyrite ore sample as a function of temperature as well as the results achieved after irradiating a Pyritebased mineral sample from two Colombian mines. At first, a brief description of microwave heating, traditional mineral recovery, and microwave assisted processing is presented. Afterwards, some experimental results are shown and main conclusions are laid out at the end.

\section{Materials and Methods}

\subsection{Microwave Heating}

Microwaves refer to electromagnetic waves whose wavelength is not in the order of micrometers, but in the range from $1 \mathrm{~mm}$ up to $1 \mathrm{~m}$. Even so, their name was assigned long ago and it has been historically preserved [15]. Frequencies associated to microwaves range from $300 \mathrm{MHz}$ up to $300 \mathrm{GHz}$. To avoid interfering with other equipment and with communications, the US Federal Communications Commission (FCC) defined some bands, known as the ISM bands. The most popular frequency band for domestic use is $2.45 \mathrm{GHz} \pm 50 \mathrm{MHz}$, though there are some industrial applicators that operate at $915 \mathrm{MHz} \pm 13 \mathrm{MHz}$ [16]. 
Microwaves can be used in different industrial scenarios, including mineral treatment. Some of the features favoring this approach are: penetrating radiation, controllable electric field distribution, rapid heating, selective heating of materials through differential absorption, and self-limiting reaction [17]. Most of the experimental results reported in literature use domestic ovens, perhaps because it represents an easy-to-access alternative. This kind of ovens generally use a magnetron to generate microwaves of low to medium power. Then, a short waveguide directs them into a rectangular cavity (or applicator) where the load (also known as MUT) is located. Within the cavity, the electromagnetic field behaves according to Maxwell's equations. In a general sense, these equations have infinite solutions, and each one is known as a propagation mode. In practice, only some of these modes are present, depending on the frequency of the wave, as well as on the dimensions of the cavity and the electromagnetic properties of the MUT. Therefore, it is possible to find applicators where one or several modes propagate, and they are known as single mode and multimode applicators, respectively. However, and since domestic microwave ovens are designed to be used with a broad ranges of elements (typically food), it is nowadays common to find multimode applicators. Furthermore, these ovens may incorporate a rotating metallic device for redirecting the waves (known as mode stirrer), striving to produce a more uniform heating in the long run.

A material, exposed to microwaves, heats because of the interaction between the alternating electromagnetic field and the inner structure of the material (whether it is a polar molecule or an electrically charged ion). This interaction turns into a translation, a rotation, or a vibration of molecules. Since several movements occur in a short period of time (due to the high frequency of the wave), the accumulated effect is an increased temperature in the sample [18]. In fact, radiation absorption at microwave frequencies encompasses phenomena such as induced electronic, atomic, and space charge polarizations.

Unlike other types of radiation (i.e. X-rays), microwaves do not possess enough energy to damage the internal structure of an element. So, as long as temperature increment is kept within a safe zone (i.e. a region where the temperature does not provoke a change of phase or a similar effect), the MUT should return to its original state after microwaves have ceased. Estimating the interaction between microwaves and a given material requires knowing the dielectric properties, i.e. its complex electrical permittivity $\left(\epsilon^{*}\right)$. This permittivity relates to the dielectric constant $\left(\epsilon^{\prime}\right)$ and to the loss factor ( $\left.\epsilon^{\prime \prime}\right)$ through eq.(1). 


$$
\epsilon^{*}(\omega ; T)=\epsilon^{\prime}(\omega ; T)+j \epsilon^{\prime \prime}(\omega ; T)
$$

The first term, on the right side, gives an estimate of how well the material absorbs electromagnetic energy. The second one refers to the ability of the material to convert energy into heat. Another parameter, which also relates to the previously mentioned components, is known as the loss tangent, $\tan \boldsymbol{\delta}$, being the quotient between the imaginary and real parts of the complex permittivity, i.e. $\tan \delta=\epsilon^{\prime \prime} / \epsilon^{\prime}$. Higher values of $\tan \delta$, electrical permittivity, and frequency, reduce the penetration depth at a particular wavelength leading to superficial heating. Lower frequencies and moderate dielectric properties lead to uniform volumetric heating. Because of the anisotropy of the Pyrite ore mineral samples, their properties are evaluated as global ones.

So far it becomes evident that, in most cases (except for electromagnetic absorbers), the material heats because of its interaction with the electric field, i.e. assuming a very low value of the complex magnetic permeability, or that the material is a non-magnetic media. As a result of that interaction, temperature increases inside the mineral sample due to the absorption of microwaves. The temperature delta can be evaluated using eq. (2), where $f$ is the frequency of the microwaves, $\varepsilon_{0}$ is the vacuum permittivity (a constant), $\rho$ the density of the sample, and $c_{p}$ its heat capacity. $E$ stands for the intensity of the electric field and $\Delta t$ for the total radiation time.

$$
\Delta T=\left[2 \pi f \varepsilon 0 \varepsilon^{\prime \prime}(\omega ; T) \rho^{-1} c_{p}^{-1 \mid}|E|^{2}\right] \Delta t
$$

\subsection{Traditional Processing}

In order to determine the Gold grade of a mineral ore, two processes are commonly carried out at laboratory level. The first one is roasting of the sample, which is done to transform sulfur-based compounds inside the ore into oxide-based ones. This process requires increasing the temperature within the sample to about $700{ }^{\circ} \mathrm{C}$, maintaining it for about $20 \mathrm{~min}$. After that, it needs to be removed from the furnace and air cooled. Finally, it is reheated for another $20 \mathrm{~min}$. The whole process takes about $160 \mathrm{~min}$, including the time needed by the furnace to increase its temperature. As a second stage, the roasted sample is smelted. This requires mixing the mineral with a set of reagents. The mixture is then heated at $950{ }^{\circ} \mathrm{C}$. Afterwards, acids are used to separate Gold from other elements. It is important to note that even though this process is supposed to yield $100 \%$ recovery, its performance can be hin- 
dered by an inappropriate roasting of the sample, although this is generally avoided by the dual heating cycle.

\subsection{Microwave Enhanced Mineral Processing}

As shown by [15], microwaves have been successfully applied in different fields. Most of them have yielded great results (e.g. reductions over $70 \%$ in processing time or in energy consumption). In the case of minerals, there is evidence of improved processing on, even, a double refractory ore (the hardest one to process) [19]. Also, studies show that microwaves generate a thermal stress inside the mineral sample, and thus create micro cracks throughout the mineral and along its boundaries. The effect is a reduced difficulty for grinding minerals by traditional means (e.g. a mill) and an increased capacity for absorbing leaching reagents, hence improving the recovery rate of the mineral [10], [20], [21].

Amaya et al. have previously obtained experimental results regarding microwave processing of Gold ores [22]. However, each ore has a varying composition and different dielectric properties, so it is necessary to explore whether the process can be replicated with a different sample. Thus, the current work furthers their research, by still considering Pyrite ore minerals (since they are difficult to process with traditional means). But, focus is given to the influence of different factors over an ore from a different mine, striving to observe if microwave benefits depend on the sample itself, or on the Pyrite content of the mineral.

Two different samples were considered for this study: one from a mine known as "Juan Blanco" (Southern Bolivar, Colombia), and one from a mine known as "Reina de Oro" (Santander, Colombia). In the first case, temperature dependence of the dielectric properties was analyzed. In the second case, a statistical experiment design was carried out to find a combination of parameters that allowed an appropriate interaction with microwaves. On this regard, the sample location inside the oven (centered), as well as the sample's mass (30 g), the grain size (sieve 100), and the output power of the oven ( $1 \mathrm{~kW})$, were constant.

Variables for this study included the sample holder, the exposure time, and the presence of a thermal enhancer, and all tests were run in random order. In the case of the first variable, four sample holders were considered: two made of clay, one made of silicon carbide, and one made of graphite. The remaining variables considered two levels each: exposure times of 24 and $36 \mathrm{~min}$, split in cycles of 6 min (manually mixing between cycles); and addition of 0 or $12 \mathrm{~g}$ of 
silicon carbide. Hence, an experimental design of four blocks was carried out, where each block contained two variables with two levels each. Three repetitions of each experiment were carried out, and a total mass of $1440 \mathrm{~g}$ was required to run all tests. Statgraphics Centurion XVII (Statpoint Technologies, Inc. - USA) was used for calculations, using a test license. As it was mentioned previously, it is paramount to analyze the evolution of complex electrical permittivity as temperature varies. Traditionally, the output of a vector network analyzer (VNA) is amplified to heat the MUT and the complex electrical permittivity is obtained by measuring the transmission coefficient. Simultaneously, temperature on the MUT is read using an infrared thermometer.

\subsection{Complex Permittivity Determination}

Values of complex electrical permittivity were obtained as a function of temperature, using a dielectric kit (DK) and a cylindrical cavity (CC), both developed at the ITACA institute (Universitat Politècnica de València, Spain). Both approaches are based on perturbation theory to estimate the appropriate parameters of a load. The first one is able to calculate the complex permittivity of a sample located inside a vial, at a given temperature, and can be used for measuring powders, liquids, and solids (provided that they are machined to match the shape of the vial) [23]. The second one is able to measure the resonant frequency $(f)$ and the quality factor $(Q)$ of a cylindrical cavity in real time, while heating the sample, and it is similar to the one developed in [24]. Combining this information, and using resonant frequency and quality factor readings for air, interpolations can be calculated and plots can be generated for each mineral, as it will be described below.

Different experimental correlations with variable orders were tested for each data set (Table 1). A search was then made for the order where the R-Square $\left(R^{2}\right)$, sum of squares due to error (SSE), and the root mean squared error (RMSE) values stabilized. As an example, consider the data shown in Figure 1. In the case of the Fourier fit all responses tSend to stabilize at the sixth and fifth orders (for heating and cooling, respectively) while the Gaussian fit stabilizes at the fifth and fourth ones (respectively), as shown in eq. 3. 
Table 1. Proposed empirical correlations of order

\begin{tabular}{|l|l|l|}
\hline \multicolumn{1}{|c|}{ Fit Name } & \multicolumn{1}{|c|}{ General Expression } & \\
\hline Fourier & $a_{0}+\sum_{i=1}^{N} a_{i} \bullet \cos (i \bullet x \bullet w)+b_{i} \bullet \sin (i \bullet x \bullet w)$ & (5) \\
\hline Gaussian & $\sum_{i=1}^{N} a_{i} \bullet e^{-\left(\frac{x-b_{i}}{c_{i}}\right)^{2}}$ & (6) \\
\hline Polynomial & $\sum_{i=1}^{N+1} p_{i} \bullet x^{N+1-i}$ & (7) \\
\hline Exponential & $\sum_{i=1}^{N} a_{i} \bullet e^{b_{i} \bullet x}$ & (8) \\
\hline Sum of Sin & $\sum_{i=1}^{N} a_{i} \bullet \sin \left(b_{i} \bullet x+c_{i}\right)$ & (9) \\
\hline
\end{tabular}

Source: author's own elaboration

Figure 1. RMSE as a function of the order used for the fitting correlation

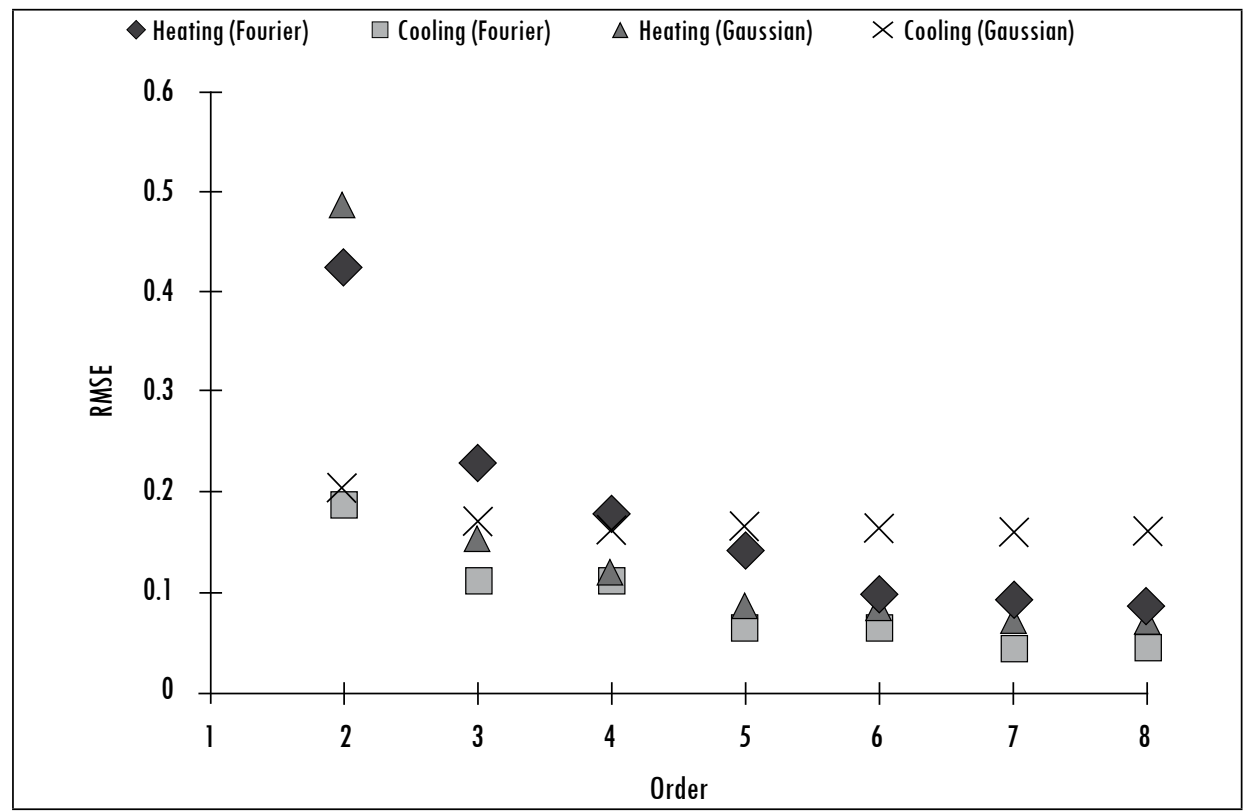

Source: author's own elaboration 


$$
\begin{aligned}
& f_{\text {heat }}(T)=2137 e^{-\left(\frac{T+6074}{54222}\right)^{2}}-10 e^{-\left(\frac{T-647}{49}\right)^{2}}-91632 e^{-\left(\frac{T-525}{69}\right)^{2}} \\
& +91623 e^{-\left(\frac{T-525}{69}\right)^{2}}-3 e^{-\left(\frac{T-410}{40}\right)^{2}} \\
& Q_{\text {heat }}(T)=1956 e^{-\left(\frac{T-11}{51}\right)^{2}}-211 e^{-\left(\frac{T-122}{89}\right)^{2}}-224 e^{-\left(\frac{T-135}{25}\right)^{2}} \\
& +542 e^{-\left(\frac{T-355}{714}\right)^{2}}
\end{aligned}
$$

In eq. (3), $f_{\text {beat }}(T)$ means the resonant frequency as a function of temperature during the heating process while $Q_{\text {beat }}(T)$ is the quality factor during the same process. Small improvements could be obtained by further increasing the orders, but it was decided to discard them to keep the fits as simple as possible. After using this experimental correlation, plots such as the one shown in Figure 2 can be generated by mapping the experimental values of the resonant frequency and quality factor, into values of complex electrical permittivity, using known data. A quadratic interpolation was considered using the resonant frequency and quality factor for air readings (2124.9 MHz and 10076, respectively), and the dielectric properties given by the dielectric kit at the beginning and at the end of the test.

Figure 2. Real (left) and imaginary (right) components of the complex permittivity for "Juan Blanco" sieve: 100 , as a function of temperature and time



Source: author's own elaboration 
As an illustrative example, consider the data given for Juan Blanco, sieve 100. The dielectric kit yields a starting complex permittivity with a real part of 5.19 and an imaginary part of 0.8818 . The reported ending values are 7.92 and 1.0925 , respectively. They correspond to initial frequency and quality factor readings of $2110.26 \mathrm{MHz}$ and 2901.14 , and final ones of $2100.43 \mathrm{MHz}$ and 1056.17 , in that order. A quadratic interpolation of these values yield eq. (4), and allows calculating the values of $\epsilon$ ' and $\epsilon$ " for any intermediate configuration of resonant frequency and quality factor.

$$
\begin{aligned}
& \epsilon^{\prime}(f)=-0.34 \times 10^{-3} f^{2}+1.16 f-917.61 \\
& \epsilon^{\prime \prime}(Q)=-0.96 \times 10^{-9} Q^{2}-0.11 \times 10^{-3} Q+1.21
\end{aligned}
$$

Figure 2 shows an evident effect of temperature and time on the dielectric properties. Still, there are some regions where the temperature remained constant for a given time, showing that the sample remained unaltered and that a thermal equilibrium was reached. In order to increase temperature further, it was required to augment the amount of power delivered to the sample. This kind of plot can become awkward and perspective may be easily lost. So, for simplicity the following data are represented focusing only on their temperature dependence.

Regarding the ore from the mine known as "Reina de Oro," a concentrated sample with a grade of $46 \mathrm{~g}$ of gold per ton of ore, was selected. In order to measure temperature, a type $\mathrm{K}$ thermocouple was used, together with the processing unit of an electric furnace, allowing real time visualization. The probe was inserted through a properly isolated perforation on the top of the microwave applicator. Also, preliminary testing showed that when using a firebrick $\left(\epsilon^{\prime}=\right.$ 2.66; $\epsilon "=0.0081)$ to isolate the sample, temperature rose an additional $100{ }^{\circ} \mathrm{C}$. Thus, the firebrick was included to focus the heat on the mineral whilst allowing microwaves to pass with low energy losses. The effects of three different factors were also investigated: the sample holder, the presence of Silicon Carbide $(\mathrm{SiC})$ on the sample and the exposure time. Four different sample holders were selected, whilst only two levels were defined for the remaining factors.

Thus, a statistical experiment designed by blocks was implemented, where each one of them related to a different sample holder, namely two clay-based (one rounded and the other one conical), one made of SiC (conical) and one made of graphite (conical). In the case of the thermal enhancer, the levels were defined as present or not, which means that a fixed amount (12 g) was used for the high level of the experiment, while no $\mathrm{SiC}$ was used in the remaining case. Finally, 
time levels were fixed at 24 and 36 min of exposure, since preliminary testing revealed that they allowed roasting the sample, while avoiding its sintering. As shown by preliminary experiments, firebricks were microwave transparent.

\section{Results}

Results from "Juan Blanco" sample revealed that the ore successfully roasted during the test when using a sieve size of 100 . Dielectric properties varied with temperature in a direct manner, shifting the resonant frequency from $2110.26 \mathrm{MHz}$ to $2100.43 \mathrm{MHz}$, and the quality factor from 2901.14 to 1056.17. The dielectric constant and loss factor increased by $52 \%$ and $24 \%$, respectively. Moreover, there was a boom for dielectric constant around $350{ }^{\circ} \mathrm{C}$ and for loss factor around $120^{\circ} \mathrm{C}$. Figure 3 shows this behavior. Arrows represent the thermal process direction (heating or cooling) in which the parameters $\left(\epsilon^{\prime}, \epsilon\right.$ " were measured. As it can be observed from this figure, there is a clear presence of thermal hysteresis. This is due to the transformation of sulfides into oxides, derived from the temperature increment. The change also implies a new internal structure for the sample. Thus, the difference in dielectric properties. Furthermore, new properties yield a different field distribution within the resonator, as well as a different level of interaction with microwaves. After the mineral has been completely roasted (highest temperature) and begins cooling, its composition is different from the starting one. Consequently, the ending dielectric properties will be different (hence, the previously mentioned hysteresis).

Now, when considering a sieve size of -200 (particle size less than $75 \mu \mathrm{m}$ ), the ore remained almost unaffected (the only change was due to evaporation of sample humidity) and the dielectric constant and loss factor decreased by $13 \%$ and $51 \%$, respectively (see Figure 4). Thus, and considering that sieve: $100 \mathrm{had}$ a sulfur content of $10.72 \%$ while sieve: -200 had only $4.27 \%$, this reduction in the affinity of the sample to microwaves may be associated with the decreasing concentration of Sulfur as the mineral is more finely ground. Figure 4 also presents the same hysteresis behavior as Figure 3. But, this time hysteresis derives from the evaporation of the sample's humidity, and so its interaction with microwaves worsens. Arrows have the same physical meaning as before. It is worth noting too that the variation of dielectric properties with temperature is fundamentally non-linear, as shown in both figures.

Table 2 shows a typical composition, determined by XRD, for a mineral sample of "Juan Blanco". As observed, there is a sharp contrast in composition before and after roasting. Pyrite and Mackinawita decreased, while Moscovite 
increased and simultaneously Spinel and Melanterite were produced. The reaction generated sulfur oxides. Table 3 shows, as an example, the coefficients of the best fit found for this sample, whilst eq. (3) showed an illustrative case of how these coefficients are reflected on the general expressions laid out in Table 1.

Table 2. Mineral composition before and after roasting for "Juan Blanco" (w\%), sieve: 100

\begin{tabular}{|l|c|c|}
\hline \multicolumn{1}{|c|}{ Compound } & Before (w\%) & After \\
\hline Quartz $\left(\mathrm{SiO}_{2}\right)$ & 18.1 & 14.0 \\
\hline Moscovite $\left(\mathrm{KAl}_{2}(\mathrm{Si}, \mathrm{Al})_{4} \mathrm{O}_{10}(\mathrm{OH})_{2}\right)$ & 17.5 & 20.1 \\
\hline Pyrite $\left(\mathrm{FeS}_{2}\right)$ & 26.7 & 12.8 \\
\hline Mackinawita $(\mathrm{FeS})$ & 2.2 & 1.5 \\
\hline Galena $(\mathrm{PbS})$ & $<1.0$ & $<1.0$ \\
\hline Arsenopyrite $(\mathrm{FeAsS})$ & N.Q. & -- \\
\hline Anglesite $\left(\mathrm{Pb}\left(\mathrm{SO}_{4}\right)\right)$ & 3.1 & -- \\
\hline Sphalerite $(\mathrm{ZnO})$ & -- & $<1.0$ \\
\hline Pyrrhotite $\left(\mathrm{Fe}_{0.95} \mathrm{~S}_{1.05}\right)$ & -- & 1.4 \\
\hline Spinel $\left(\mathrm{Mg}_{1.01} \mathrm{Fe}_{1.77} \mathrm{Al}{ }_{.22} \mathrm{O}_{4}\right)$ & -- & 1.9 \\
\hline Melanterite $\left(\left(\mathrm{Fe}, \mathrm{Cu}_{1} \mathrm{Zn}_{2}\right) \mathrm{SO}_{4} \cdot 7 \mathrm{H}_{2} \mathrm{O}\right)$ & -- & 1.9 \\
\hline Zincocopiapite $\left(\mathrm{ZnFe}{ }_{4}\left(\mathrm{SO}_{4}\right)_{6}(\mathrm{OH}) 2 \cdot 18 \mathrm{H}_{2} \mathrm{O}\right)$ & -- & N.Q. \\
\hline Anatase $\left(\mathrm{TiO}_{2}\right)$ & -- & $<1.0$ \\
\hline Others & 31.6 & 44.3 \\
\hline
\end{tabular}

N.Q.: Not Quantifiable

Source: author's own elaboration

Table 3. Summary of best fits for the mineral sample "Juan Blanco," sieve: 100. The general expression of each model is shown in Table 1. Starting dielectric properties are: $\epsilon^{\prime}=5.19$; $\epsilon^{\prime \prime}=0.8818$. Ending dielectric properties are: $\epsilon^{\prime}=7.92 ; \epsilon^{\prime \prime}=1.0925$

\begin{tabular}{|c|l|l|l|}
\hline Process & \multicolumn{1}{|c|}{ Suggested Fit } & \multicolumn{2}{c|}{ Coefficients } \\
\hline Heating & Frequency: & ai $=$ & {$[2137,-9.92,-91632.63,91623.18,-2.84]$} \\
\hline & Gaussian N=5 & bi $=$ & {$[-6074,647,525.30,525.30,409.90]$} \\
\hline
\end{tabular}




\begin{tabular}{|l|l|l|l|}
\hline Process & \multicolumn{1}{|c|}{ Suggested Fit } & \multicolumn{1}{|c|}{ Coefficients } \\
\hline & $R^{2}=0.9998$ & $\mathrm{ci}=$ & {$[54221.56,49.13,69.19,69.19,39.37]$} \\
\hline Cooling & Gaussian $\mathrm{N}=4$ & $\mathrm{ai}=$ & {$[2100,40611.59,4.96,0.74]$} \\
\hline & $R^{2}=0.9970$ & $\mathrm{bi}=$ & {$[-7.17,1272,578.20,458]$} \\
\hline & & $\mathrm{ci}=$ & {$[6733,216.20,90.81,458]$} \\
\hline Heating & Quality Factor: & $\mathrm{ai}=$ & {$[1956,2116,-224.20,541.90]$} \\
\hline & Gaussian $\mathrm{N}=4$ & $\mathrm{bi}=$ & {$[10.83,121.80,135,355.40]$} \\
\hline & $R^{2}=0.9983$ & $\mathrm{ci}=$ & {$[51.03,89.29,25.28,713.90]$} \\
\hline Cooling & Gaussian $\mathrm{N}=5$ & $\mathrm{ai}=$ & {$[-96.23,-41157.72,41281.25,-93.43,1568]$} \\
\hline & $R^{2}=0.9955$ & $\mathrm{bi}=$ & {$[-10.24,168.90,168.90,456.90,-587.80]$} \\
\hline & & $\mathrm{ci}=$ & {$[115.60,74.05,74.24,83.89,1040]$} \\
\hline
\end{tabular}

Source: author's own elaboration

Figure 3. Dielectric properties as a function of temperature for "Juan Blanco," sieve: 100

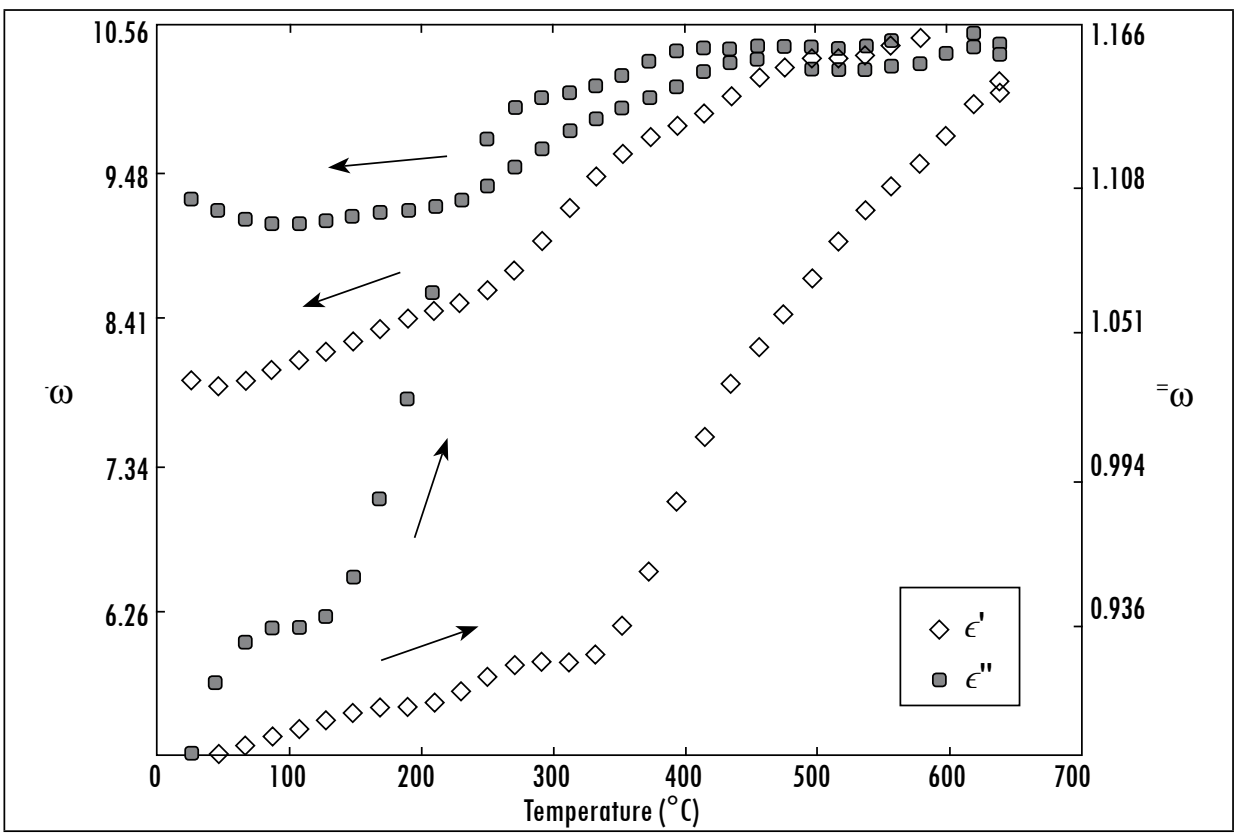

Note. The black, empty diamond represents the real part of the permittivity. The gray, filled square represents the complex part of the permittivity.

Source: author's own elaboration 
Figure 4. Dielectric properties as a function of temperature for "Juan Blanco," sieve: -200

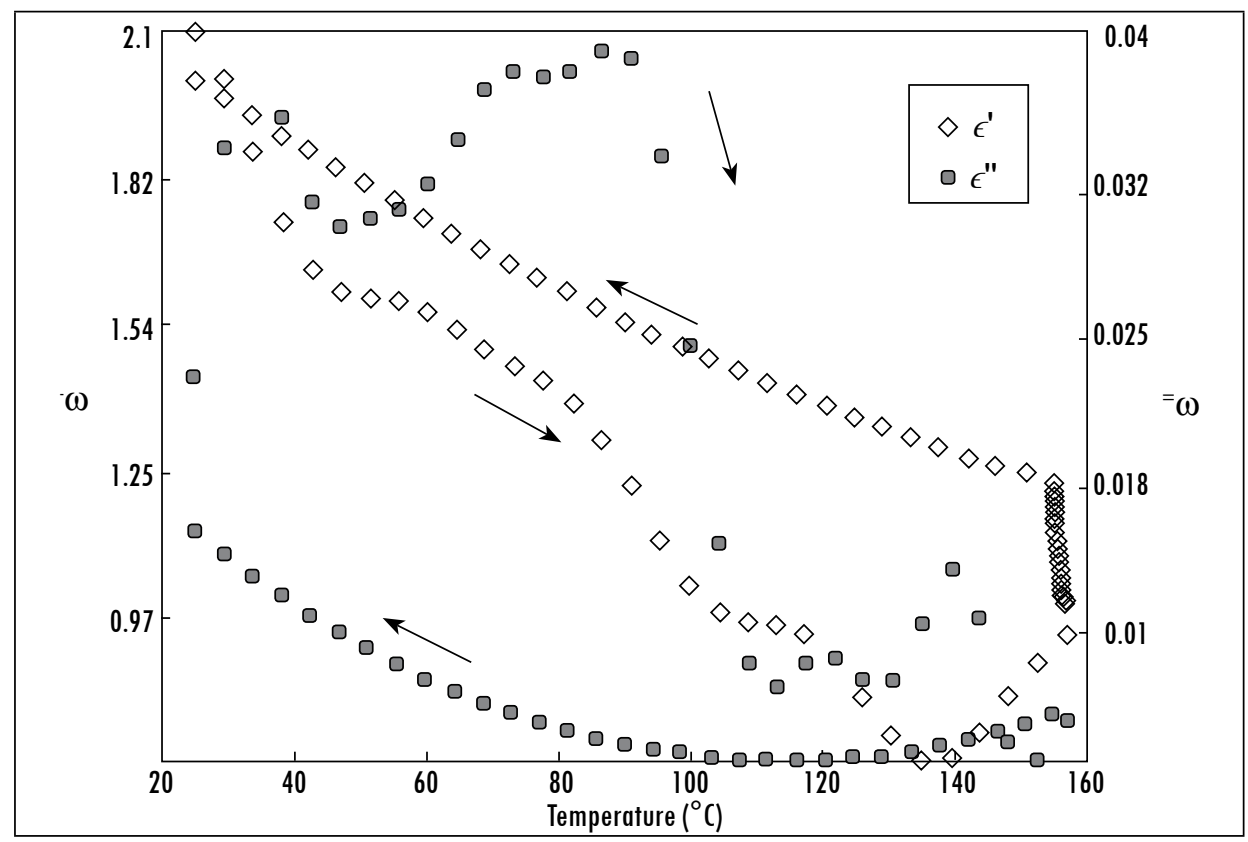

Note. The black, empty diamond represents the real part of the permittivity. The gray, filled square represents the complex part of the permittivity.

Source: author's own elaboration

Opposed to expectations, using a thermal enhancer not always yielded positive effects. This is shown by the last column of Table 4, where a lower grade was obtained when considering the presence of $\mathrm{SiC}$ and using the graphite sample-holder. In order to explore this effect further, a test was run, measuring temperature for $48 \mathrm{~min}$, with and without enhancer, and for all sample-holders. Figure 5 shows the second case, and it as can be observed, the lossier behavior of Graphite and of $\mathrm{SiC}$ generate an increase in the sample's temperature. On the other hand, Figure 6 shows temperature evolution when using the enhancer. In this case, however, the behavior for $\mathrm{SiC}$ and Graphite sample-holders becomes somewhat more erratic. This phenomenon can be explained by a race condition, where thermal enhancer and sample holder compete for the electric field. Throughout all the experiments, firebricks were used to avoid loss of thermal energy. 
Table 4. Grade $(\mathrm{g} / \mathrm{t})$ of microwave roasted "Reina de Oro" samples

\begin{tabular}{|c|c|c|c|c|c|c|c|c|c|}
\hline \multirow{3}{*}{$\begin{array}{c}\text { Time } \\
\text { (min) }\end{array}$} & \multicolumn{7}{|c|}{ SiC } \\
\cline { 2 - 10 } & \multicolumn{7}{|c|}{ No } & \multicolumn{7}{c|}{ Yample holder } \\
\cline { 2 - 10 } & $\mathbf{1}$ & $\mathbf{2}$ & $\mathbf{3}$ & $\mathbf{4}$ & $\mathbf{1}$ & $\mathbf{2}$ & 3 & 4 \\
\hline \multirow{3}{*}{24} & 1.0 & 0.0 & 17.0 & 24.7 & & 9.3 & 50.0 & 20.7 & 4.3 \\
\cline { 2 - 10 } & 2.3 & 0.7 & 22.3 & 30.1 & & 10.4 & 45.0 & 19.7 & 12.3 \\
\cline { 2 - 10 } & 1.9 & 0.9 & 20.3 & 28.1 & & 8.3 & 40.0 & 18.6 & 7.8 \\
\hline \multirow{3}{*}{36} & 6.7 & 0.7 & 20.0 & 52.0 & & 39.3 & 54.7 & 36.3 & 20.0 \\
\cline { 2 - 10 } & 7.0 & 1.0 & 23.4 & 40.0 & & 38.2 & 47.2 & 36.7 & 18.7 \\
\cline { 2 - 10 } & 5.3 & 1.1 & 23.4 & 45.1 & & 35.1 & 43.3 & 34.3 & 19.3 \\
\hline
\end{tabular}

Source: author's own elaboration

Figure 5. Temperature measurements for all sample holders and no thermal enhancer, using "Reina de Oro" samples

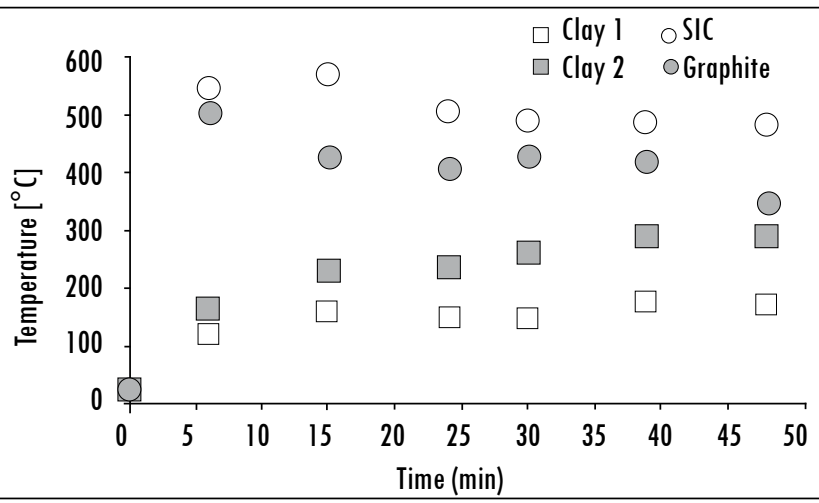

Source: author's own elaboration 
Figure 6. Temperature measurements for all sample holders and thermal enhancer, using "Reina de Oro" samples

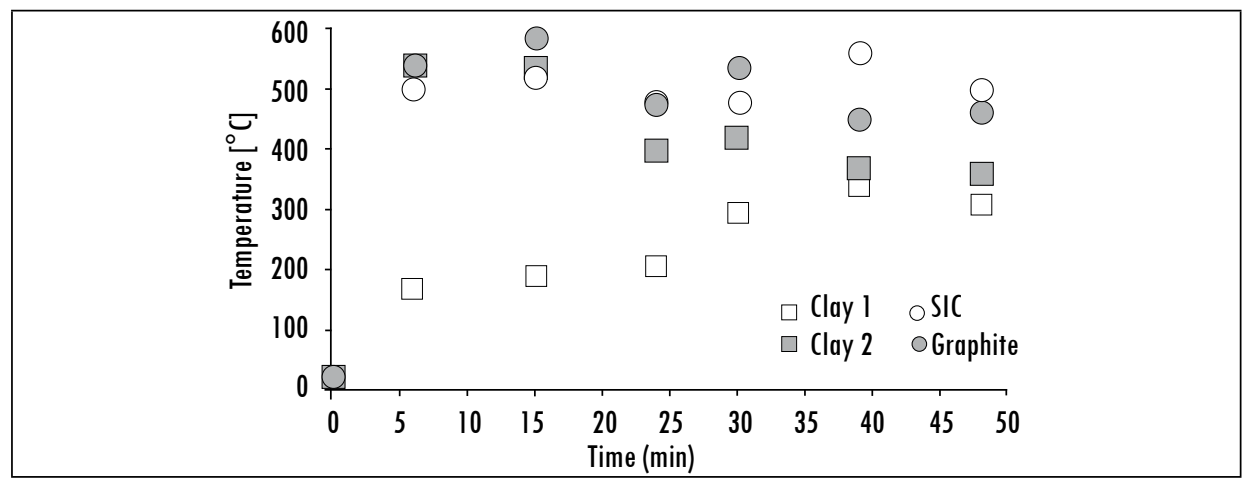

Source: author's own elaboration

\subsection{Preliminary Smelting Tests}

The aforementioned data motivated a preliminary smelting test. Three samples were used, one roasted by traditional means and two roasted by microwaves. At least for these preliminary tests, using microwaves allows for a proper smelting of the mineral ores (Table 5). Two things are worth mentioning here. The first one is that reagents are still used to facilitate the smelting process, so there is no gain in this sense (however, the reagents used for smelting at lab level are not as toxic and/or dangerous as the ones used for leaching ores).

Table 5. Preliminary results of microwave smelted "Reina de Oro" samples

\begin{tabular}{|l|c|c|c|}
\hline \multicolumn{1}{|c|}{ Sample } & $\begin{array}{c}\text { Grade } \\
\text { (microwave) }(\mathbf{g} / \mathbf{t})\end{array}$ & $\begin{array}{c}\text { Grade } \\
\text { (traditional) } \mathbf{( g / t )}\end{array}$ & $\begin{array}{c}\text { Recovery } \\
\text { margin }(\%)\end{array}$ \\
\hline Traditionally roasted & 43.7 & 46.0 & 95 \\
\hline $\begin{array}{l}\text { Roasted using SiC sample-holder and } \\
\text { thermal enhancer }\end{array}$ & 36.7 & 36.7 & 100 \\
\hline $\begin{array}{l}\text { Roasted using graphite sample- } \\
\text { holder and no thermal enhancer }\end{array}$ & 40.0 & 40.0 & 100 \\
\hline
\end{tabular}

Source: author's own elaboration

The second remark is that the whole smelting process (starting at ambient temperature) only took $20 \mathrm{~min}$, as opposed to approximate four hours required by traditional smelting in an electric furnace. So, a reduction in processing time of about $92 \%$ can be easily achieved. Thus, the economic and environmental benefits of microwave enhanced processing are enhanced even further. Even 
though an energy reading was not registered for microwave smelting (mainly due to the exploratory nature of the tests), it is fairly obvious that an energy reduction in the order of the one achieved for roasting (or even greater) can be obtained.

\section{Conclusions}

We showed an experimental analysis related to measuring dielectric properties of Pyrite ore mineral samples as a function of temperature, as well as the effect of those properties on their ability to absorb microwave energy.

Dielectric properties of the samples are highly dependent on temperature. Some empirical correlations for predicting their values were proposed, valid within the limiting temperature range. The nonlinear behavior exhibited by the samples is likely due to the change in composition of the samples, since new compounds were formed by oxidation and others were eliminated during heating. For that reason, it is of particular importance to include the variation of this parameters with temperature, an aspect not included in most of the research results reported dealing with this kind of mineral ores.

As can be seen in Figure 2, Figure 3, and Figure 4, throughout the studied temperature range both components of the complex permittivity ( $\epsilon^{\prime}$ and $\left.\epsilon^{\prime \prime}\right)$ present a behavior quite similar to thermal hysteresis. Furthermore, a relationship between grain size and microwave interaction was found. If the grain is too small, there is almost no heating of the ore. Microwave processing of materials could be predicted and controlled in real time, should the mineral composition be determined instantly, since microwaves selectively heat the material with higher loss factor.

Microwaves allowed for a higher recovery rate than traditional means when roasting a pyritic mineral sample at lab-scale. The best scenario found throughout this research requires using a firebrick, a clay-based sample holder, with $\mathrm{SiC}$ as a thermal enhancer and a processing time of $36 \mathrm{~min}$. Under these conditions, less processing time and energy were required (about $77.5 \%$ and $80.8 \%$ less, respectively). The aforementioned phenomenon can be explained by a direct transfer of energy to the mineral, eliminating heat losses associated to furnaces and their walls, as well as to heat transfer media. Thus, higher heat transfer rates than with the conventional method can be achieved, yielding higher temperatures in shorter times.

An exploratory test, carried out in order to assess the feasibility of using microwaves to smelt a roasted mineral ore, showed that time reductions of about 
92\% are possible. Recovery margins were located between $95 \%$ and $100 \%$ when compared to traditional smelting. On this regard, it was also found that microwave roasted samples yielded better recovery margins (i.e. they were the ones that allowed a total recovery). This could be caused by the increased thermal stress generated by microwaves.

In spite of the benefits, the results shown throughout this manuscript have some limitations, so it is important to address them. On this regard, the first thing to mention is that a limited number of ores were used. This, however, was done because research focused on strengthening previously reported results with similar compounds, testing other mineral ores, and different design variables. Moreover, it would be impractical to pretend considering all possible combinations of mineral ores. Furthermore, analytic tests were carried out to verify the composition of the ores, finding that Pyrite seems to be the main element that allows for a proper interaction with microwaves. Hence, samples with similar concentrations should exhibit a similar behavior. Another limitation of the data is that they were obtained at lab scale. Microwaves are not scalable by nature, so this inhibits a direct scale up to pilot plant level. Still, it is important to first assess the possible benefit that may derive from an alternate procedure such as microwave roasting (and smelting), in order to justify the increased expenses of implementing it at a higher scale. Finally, just some tests were executed regarding microwave smelting. This was done because the nature of the tests were exploratory and they were inspired on the good results obtained for microwave roasting.

Similarly, as reported by other authors, it was found that the collapse of the microwave roasted samples at lower temperatures for shorter roasting times was more extensive than that of the samples traditionally roasted at higher temperatures and even longer times. We also found that the enhanced benefit of treating samples with microwaves depends heavily on the Pyrite content of the ore. The economic and environmental implications of these results are evident, and it is recommended to scale the research, as a next step, by using a single mode applicator where the mineral can be easily placed in a given uniform zone of the electric field - e.g. in a partially filled waveguide - or by striving to process a higher volume of mineral.

\section{Acknowledgment}

The authors would like to express their gratitude to Vicerrectoría de Investigación y Extensión, at Universidad Industrial de Santander (Colombia), for 
the support granted through project 5551, and to the Institute at Universitat Politècnica de València (Spain) for the support granted regarding the measurement of dielectric properties.

\section{References}

[1] Keysight Technologies, "Application note: basics of measuring the dielectric properties of materials." Keysight Technologies, pp. 1-34, 2015.

[2] Y. Zhao, Y. Hou, Y. Cui, H. Liang, and L. Li, "Recovery of copper from copper sulfide concentrate by sulfation roasting," Int.J. Nonferrous Metall., vol. 04, no. 2, pp. 9-13, Apr. 2015.

[3] X. Ma, M. Zhang, F. Min, T. Ge, and C. Cai, "Fundamental study on removal of organic sulfur from coal by microwave irradiation," Int. J. Miner. Process., vol. 139, pp. 31-35, Jun. 2015.

[4] T. Agacayak and M. Koseler, "Effect of microwave heating on the leaching of lateritic nickel ore in perchloric acid," J. Chem. Soc. Pakistan, vol. 37, no. 2, pp. 230-235, Apr. 2015.

[5] Z. Li, J. Li, L. Zhang, J. Peng, S. Wang, A. Ma, and B. Wang, "Response surface optimization of process parameters for removal of $\mathrm{F}$ and $\mathrm{Cl}$ from zinc oxide fume by microwave roasting," Trans. Nonferrous Met. Soc. China, vol. 25, no. 3, pp. 973-980, Mar. 2015.

[6] S. Singh, D. Gupta, V. Jain, and A. Sharma, "Microwave processing of materials and applications in manufacturing industries: a review," Mater. Manuf. Process., vol. 30, no. 1, pp. 37-41, May 2015.

[7] M. Wang, P. Xian, X. Wang, and B. Li, "Extraction of vanadium from stone coal by microwave assisted sulfation roasting," JOM, vol. 67, no. 2, pp. 369-374, Feb. 2015.

[8] Z. Guo, T. Lei, W. Li, H. Luo, S. Ju, J. Peng, and L. Zhang, "Clean utilization of CuCl residue by microwave roasting under the atmosphere of steam and oxygen," Chem. Eng. Process. Process Intensif., vol. 92, pp. 67-73, Jun. 2015.

[9] K. Hara, M. Hayashi, M. Sato, and K. Nagata, "Continuous pig iron making by microwave heating with $12.5 \mathrm{~kW}$ at $2.45 \mathrm{GHz}$," J. Microw. Power Electromagn. Energy, vol. 45, no. 3, pp. 137-147, 2011.

[10] O. Peltosaari, P. Tanskanen, E.-P. Heikkinen, and T. Fabritius, " $A \rightarrow \Gamma \rightarrow B$-phase transformation of spodumene with hybrid microwave and conventional furnaces," Miner. Eng., pp. 1-7, Apr. 2015.

[11] E. R. Bobicki, Q. Liu, and Z. Xu, "Microwave heating of ultramafic nickel ores and mineralogical effects,” Miner. Eng., vol. 58, pp. 22-25, Apr. 2014.

[12] W. Zhao, J. Chen, X. Chang, S. Guo, C. Srinivasakannan, G. Chen, and J. Peng, "Effect of microwave irradiation on selective heating behavior and magnetic separation characteristics of Panzhihua ilmenite," Appl. Surf. Sci., vol. 300, pp. 171-177, May 2014. 
[13] M. Lovás, M. Kováčová, G. Dimitrakis, S. Čuvanová, I. Znamenáčková, and Š. Jakabský, "Modeling of microwave heating of andesite and minerals," Int. J. Heat Mass Transf., vol. 53, no. 17-18, pp. 3387-3393, Aug. 2010.

[14] Y. Wang and N. Djordjevic, "Thermal stress FEM analysis of rock with microwave energy," Int. J. Miner. Process, vol. 130, pp. 74-81, Jul. 2014.

[15] I. Amaya and R. Correa, "Electromagnetic heating as a way of cutting costs while saving energy: Time evolution," Rev. Ing. Univ. Medellín, vol. 11, no. 20, pp. 215-226, Jun. 2012.

[16] R. Meredith, Engineers' Handbook of Industrial Microwave Heating. London: The Institution of Electrical Engineers, 1998.

[17] X. J. Su, S. J. Ma, C. L. He, and Y. Q. Chen, "Direct microwave roasting of arsenic- bearing pyrite concentrates," J. Microw. Power Electromagn. Energy, vol. 48, no. 2, pp. 81-88, 2014.

[18] R. Correa, F. Ortiz, and R. Cruz, "Medición en línea de la temperatura de una muestra en una cavidad de microondas," Rev. Fac. Ing. Univ. Antioquia, no. 52, pp. 123-133, Mar. 2010.

[19] B. Nanthakumar, C. Pickles, and S. Kelebek, "Microwave pretreatment of a double refractory Gold ore,” Miner. Eng., vol. 20, no. 11, pp. 1109-1119, Sep. 2007.

[20] R. K. Amankwah and G. Ofori-Sarpong, "Microwave heating of gold ores for enhanced grindability and cyanide amenability," Miner. Eng., vol. 24, no. 6, pp. 541-544, May 2011.

[21] R. K. Amankwah, A. U. Khan, C. A. Pickles, and W. T. Yen, "Improved grindability and gold liberation by microwave pretreatment of a free-milling gold ore," Miner. Process. Extr. Metall., vol. 114, no. 1, pp. 30-36, Mar. 2005.

[22] I. Amaya, D. Bernal, S. Garnica, M. Reslen, and R. Correa, "Improved roasting of some Colombian gold ores," Dyna, vol. 80, no. 178, pp. 70-77, 2013.

[23] J. M. Catalá Civera, J. D. Gutiérrez Cano, F. L. Peñaranda-Foix, and B. Garcia-Baños, "Portable system for dielectric characterization of materials at microwave frequencies," in 13th International Conference on Microwave and RF heating, AMPERE 2011, 2011, pp. 137-140.

[24] A. J. Canos, F. L. Penaranda-Foix, J. M. Catala-Civera, and B. Garcia-Banos, "Measurement of dielectric properties at high-temperatures in real-time with cylindrical cavity," in 2010 IEEE MTT-S International Microwave Symposium, 2010, pp. 1044-1047. 